\title{
A System Dynamics Simulation Model of Hierarchical Medical Care System Reform in China
}

\author{
Hao Zhang, Yue Liu, Qian Yang, Shuyan Gu, Xumei Zhen, Yongpeng Xia, Jianglei Zhao, Hai Yu and Hengjin Dong* \\ Center for Health Policy Studies, Zhejiang University School of Medicine, Hangzhou, China
}

\begin{abstract}
The hierarchical medical care system reform in China may lead to drastic changes of medical care utilizations distribution in different medical care subsystems. A medical care system dynamics model was constructed to simulate the intervention scenarios according to the reform measures, and to estimate changes of demands, utilizations distribution and reasonable resources allocation, in order to provide optimized intervention for decision-makers.
\end{abstract}

\section{Keywords: Hierarchical; Community Health Systems (CHSs)}

\section{Introduction}

The Chinese government has been carrying out a new health system reform, an important task of which is to establish a reasonable hierarchical medical care system. The existing system in China is so called "hospital-centrism" system by World Health Organization, which allocates excessive resources in hospitals, over-valued specialist and tertiary care [1]. Meanwhile, there is no gatekeeper who controls patient's entrance into hospitals during the first-contact diagnosis [2]. Therefore, groups in higher socioeconomic class tend to access hospital service unreasonably while the utilization of Community Health Systems (CHSs) service is at a low level [3]. Such unbalanced resource allocation and disordered medical care procedure lead to inequity and inefficiency [4]. The government had committed to solve this problem by setting a gatekeeper in CHSs, by taking reform measures to improve CHSs primary diagnosis and service utilization in some pilot regions. For instance, Zhejiang Province, aims to provide CHSs primary diagnosis for not less than $60 \%$ of the patients by 2020 .

The reform may lead to drastic changes of medical care utilizations distribution in different medical care subsystems, that is, utilizations may increase in CHSs and decrease in hospitals. It may bring challenges to readjust resources correspondingly, especially in human resources and health funds. Therefore, reasonably forecasting changes of medical service utilizations distribution, and estimating resources allocation is valuable for decision-makers. We construct a medical care system model by System Dynamics methods (SD), simulating intervention scenarios according to the reform measures, and estimating the changes of demands, service utilizations distribution and reasonable resources allocation, in order to provide some references.

\section{Methods}

We simulate the medical care system with System Dynamics (SD) tools. Compared to a variety of other methods, the SD model offers obvious advantages. It is capable of stimulating large and complex system, since it is able to deal with high order, nonlinear, time varying function. It may show us long-term predicted reform efforts, since it is able to simulate long-term changes by setting time parameters in the future. It may provide us with optimized interventions to promote CHSs primary diagnosis, since it can set various levels of patient's visiting behaviour-influencing factors. It may improve the reliability of the model, by setting constraints according to the real world, for instance, increased population generating new demands and the available resource restricting medical service supplying [5-9]. The SD model is built with software Vensim 5.6a (USA, Ventana Systems, Inc.).
The steps involved in the simulation are: Construct a logical model by defining the system boundary, by identifying the key factors and their interactions, and by drawing causal loop diagrams aiding in visualizing the system's structure and behaviour. Construct the model in a quantitative way by drawing stock and flow model, and by writing the equations that determine the flows. Estimate the parameters and set constraints by statistical analysis of the data from real world system, for instance, the prevalence rate, consultation rate, the odd ratio of CHSs primary diagnosis choosing possibilities when adjusting the proportion of health insurance compensation [10]. Simulate the model and test the model's validation with historical data of real world system. In this model, we use the data of Zhejiang Province for quantitative analysis, since this province is one of the pilot regions. After simulating the existing disordered medical care system, we adjust some parameters to simulate the reform-intervened system, for instance, adjust the proportion of health insurance compensation. Establishing such intervention scenarios, we may simulate the medical care utilization in hospitals and CHSs, and estimate the resources correspondingly. Additionally, we set different levels of parameters to analyse the model sensitivity, and optimize the reform interventions.

\section{Results}

\section{Logical model analysis and stock and flow model illustrating}

The medical care utilizations are the core indicators of the model, because the reform's target is to establish ordered medical care procedure and to readjust utilization distribution reasonably. We construct three subsystems around the core indicators: The patient's demands subsystem, the outpatient and inpatient utilization subsystem in CHSs and hospitals, and the resources allocation subsystem. We simulate the main factors and their interactions by creating stocks, flows and internal feedback loops. A stock, the term for any entity that accumulates or depletes over time, is used to simulate the medical care demands and resources. A flow, the rate of changes in a stock, is used to simulate

*Corresponding author: Hengjin Dong, Center for Health Policy Studies, Zhejiang University School of Medicine, Hangzhou, China, Tel: +132-2107-6129; E-mail: donghj@zju.edu.cn

Received February 27, 2017; Accepted March 30, 2017; Published April 5 , 2017

Citation: Zhang H, Liu Y, Yang Q, Gu S, Zhen X, et al. (2017) A System Dynamics Simulation Model of Hierarchical Medical Care System Reform in China. J Health Med Informat 8: 253. doi: 10.4172/2157-7420.1000253

Copyright: $\odot 2017$ Zhang $\mathrm{H}$, et al. This is an open-access article distributed under the terms of the Creative Commons Attribution License, which permits unrestricted use, distribution, and reproduction in any medium, provided the original author and source are credited. 
the demands changes and the resources adjustment. Internal feedback loops is used to describe the interactions of flow-influencing factors.

\section{The patient's demands subsystem}

Model description: The patient's unreasonable demands may cause unreasonable service utilization; the reform takes measures to intervene on medical behaviour-influencing factors. Analysis of literatures and questionnaires survey data of our previous studies shown that, the patient's visiting behaviour is mainly influenced by these factors: Demographic characteristics (Gender, marriage, age, education, career etc.), economic condition (income level, consumption level, etc.), physical condition (health self-raking, chronic diseases, acute disease, etc.), medical service utilization (emergency treatment, outpatient treatment, hospitalization), service condition (technology level, medical devices and environment, responsiveness, etc.), accessibility (distance from medical care institutions, accessible transport), medical insurance (the proportion of health insurance compensation, the gap of compensable proportion of CHSs and hospitals, etc.), medical expenses (medical service price, the total treatment cost, etc.), doctor-patient relationship, and opinion of CHSs and hospitals service [11-13]. This factor may influence the key indicator: Consulting rate, that is, whether to seeking medical service or not. And so does another key indicator: Choice of primary diagnosis provider that is, seeking first-contact in CHSs or hospital. The reform aims to adjust factors above (improving the CHSs service quality, reducing service price, increasing the gap of medical insurance compensable proportion) to promote CHSs primary diagnosis and servers utilization. We adjust the levels of these factors according to the reform, and simulate patients' demands for CHSs and hospitals.

Stock and flow model: Stock-flow diagram of the patient's demands subsystem (Figure 1).

Population constraints: The population is a constraint factor of this subsystem, since the medical care needs and demands are determined by population, and the reform aims to meet the reasonable demand of the population. The indicator of population is determined by the birth rate, the fertility pattern, the mortality, the existing population, the life expectancy, the migration condition, and the sex ratio at birth. We use the Markov model to predict the population changes with these seven factors, by the forecasting software PADIS-INT [14].

The matrix of population varying in ages, sex in year $(\mathrm{t})$ and year $(\mathrm{t}-$ 1) are as follows: $P_{t}^{a, s}=\left(1-d_{t}^{a, s}\right) P_{t-1}^{a, s}+N I_{t}^{a, s}$

(Formula 1)

The new-born population is as follows:

$$
P_{t}^{0, s}=\eta^{s} \cdot\left(1-d_{t}^{0, s}\right) \cdot \sum_{15}^{49}\left(f_{t}^{a, s=1} P_{T}^{A, S=1}\right)
$$

Note: $d_{t}^{a, s}$ represents the population, $d_{t}^{a, s}$ represents the mortality rate, $N I_{t}^{a, s}$ represents infant mortality rate, $N I_{t}^{a, s}$ represents immigration population, $f_{t}^{a, s}$ represents women's fertility rate, $\eta^{s}$ represents sex ratio at birth. Above indicators are all under such circumstances: Year $=\mathrm{t}$, Gender $=\mathrm{s}(\mathrm{s}=0$ or $1,0=$ Male, $1=$ Female), age $=\mathrm{a}$.

\section{The subsystem of service utilization in hospitals and CHSs}

Model description: There are three levels of health care institutions in China: The tertiary hospitals (which are positioned to provide tertiary service for inpatients and outpatient treatment with difficult miscellaneous diseases and some acute treatment), the secondclass hospitals (which are positioned to provide specialized service for inpatients and outpatient treatment with common diseases and frequently-occurring diseases), and primary health care institutions in CHSs (which are positioned to provide general medical treatment and other primary health care services). In actuality, patients with common and frequently-occurring diseases also seek hospital primary diagnosis, and the hospitals are willing to provide overstepped service for economic benefits [15]. The reform may restrict the hospitals' service scope and promote CHSs primary diagnosis.

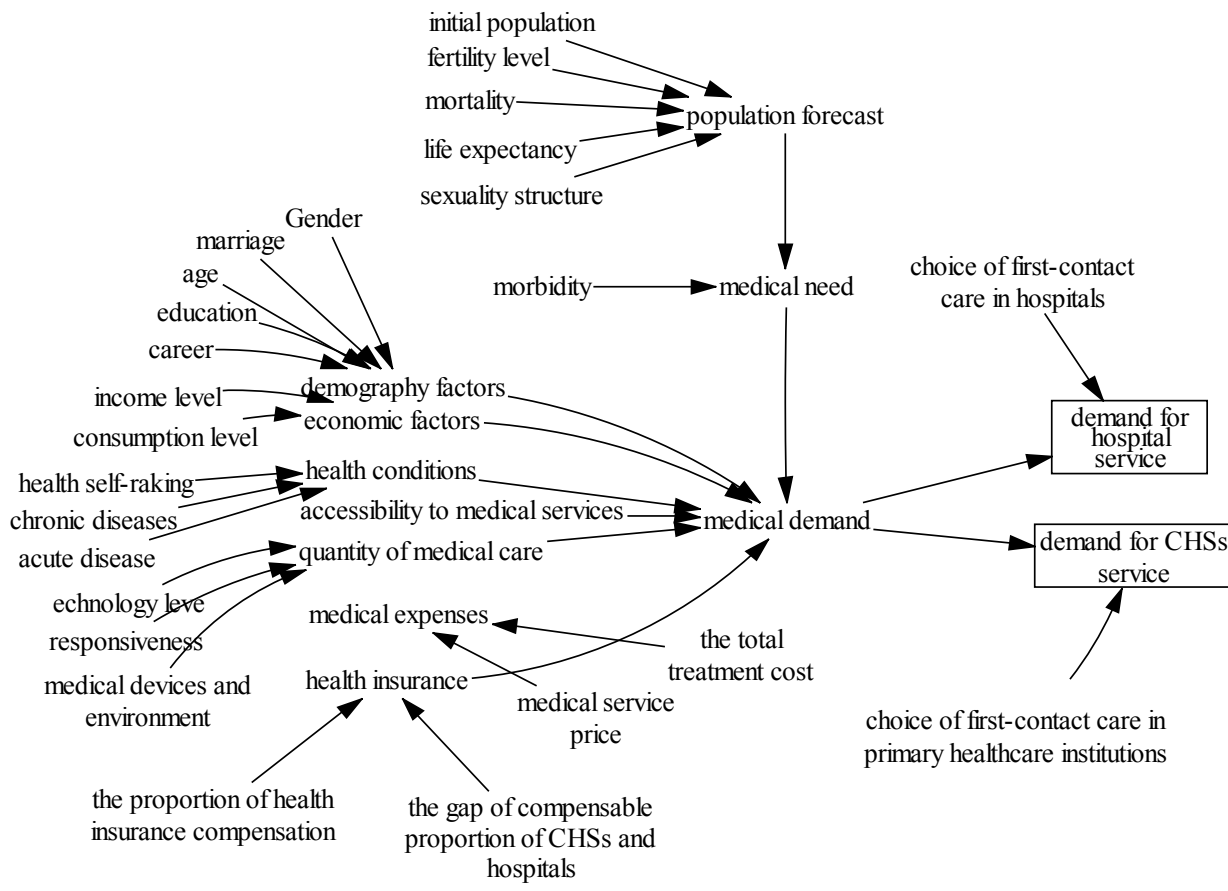

Figure 1: Stock-flow diagram of the patient's demands subsystem. 
Service utilizations in medical care institutions are simulated with a stock, accumulating by the flow of first-contract diagnosis, subsequent visiting, downwards-referral from higher level institutions and upwards-referral from lower-level institutions, and depleting by flow of patient cured, running off, deceased, upwards-referral to higher level institutions, and downwards-referral to lower-level institutions. In an ideal medical care system, patients with different degrees of disease should be referred to different levels of institution reasonably through orderly and smooth referral procedure. However, the actual downward referral rate is at a low level, since the hospitals showing little willingness to refer downwards.

Stock and flow model: Stock-flow diagram of subsystem of service utilization in hospitals and CHSs (Figure 2).

\section{Resources constraints}

Labour and capital are two main indicators determining medical service output, that is, the service utilizations are limited by resource. We use Cobb-Douglas production function to estimate the maximum service supply with existing available labour and capital [16], and set the maximum service supply as the constrains of this subsystem.

The production function is as follows:

$$
\mathrm{Y}=\mathrm{AK} \beta \mathrm{L} \alpha
$$

Note: Y represents maximum service supply, a represents multifactor productivity, $\mathrm{K}$ represents health care fund, $\mathrm{L}$ represents health care labour force, $\alpha$ and $\beta$ are the elasticity's of capital and labour, respectively.

\section{Subsystem of resources allocation}

Model description: Downward-adjusting service utilizations require downward-allocating resources. The labour force demand in CHSs may be estimated by Workload Indicators of Staffing Need method (WISN) [17]. The reform sets three channels for labour force adjustment, CHSs-oriented student education, staff recruiting and hospital labour force downward-transition. The number of current employee is simulated with a stock, accumulating by flows through the three channels mentioned above, and depleting by flow of resignation, retirement and brain drain. In this model, we may simulate labour force vacancies and estimate the labour force quantity for adjustment through the three channels, respectively.

Similarly, capital adjustment may also be simulated by the system. Thus, the direct medical costs are much lower in CHSs than in hospitals, let alone the indirect cost saved from shorter commuting, more convenient accompany, etc. The saved funds should be redistributed reasonably, an appropriate part of which should compensate the hospitals' financial lost due to the reform.

Stock and flow model: Stock-flow diagrams of human resources (Figure 3). Stock-flow diagram of capital (Figure 4).

\section{Main equations that determine the stocks and flows}

$$
L(t)=l_{0+} \int_{0}^{t}\left(\sum R_{I N}(t)\right) d t
$$

Note: $\mathrm{L}(\mathrm{t})$ represents the level of the stock at time (t), L0 represents initial level, Rin represents flow rate putting in, Rout represents flow rate putting out.

\section{Model validations}

To validate the model, we use the date from Zhejiang Province to estimate parameters, and then simulate the model for five years

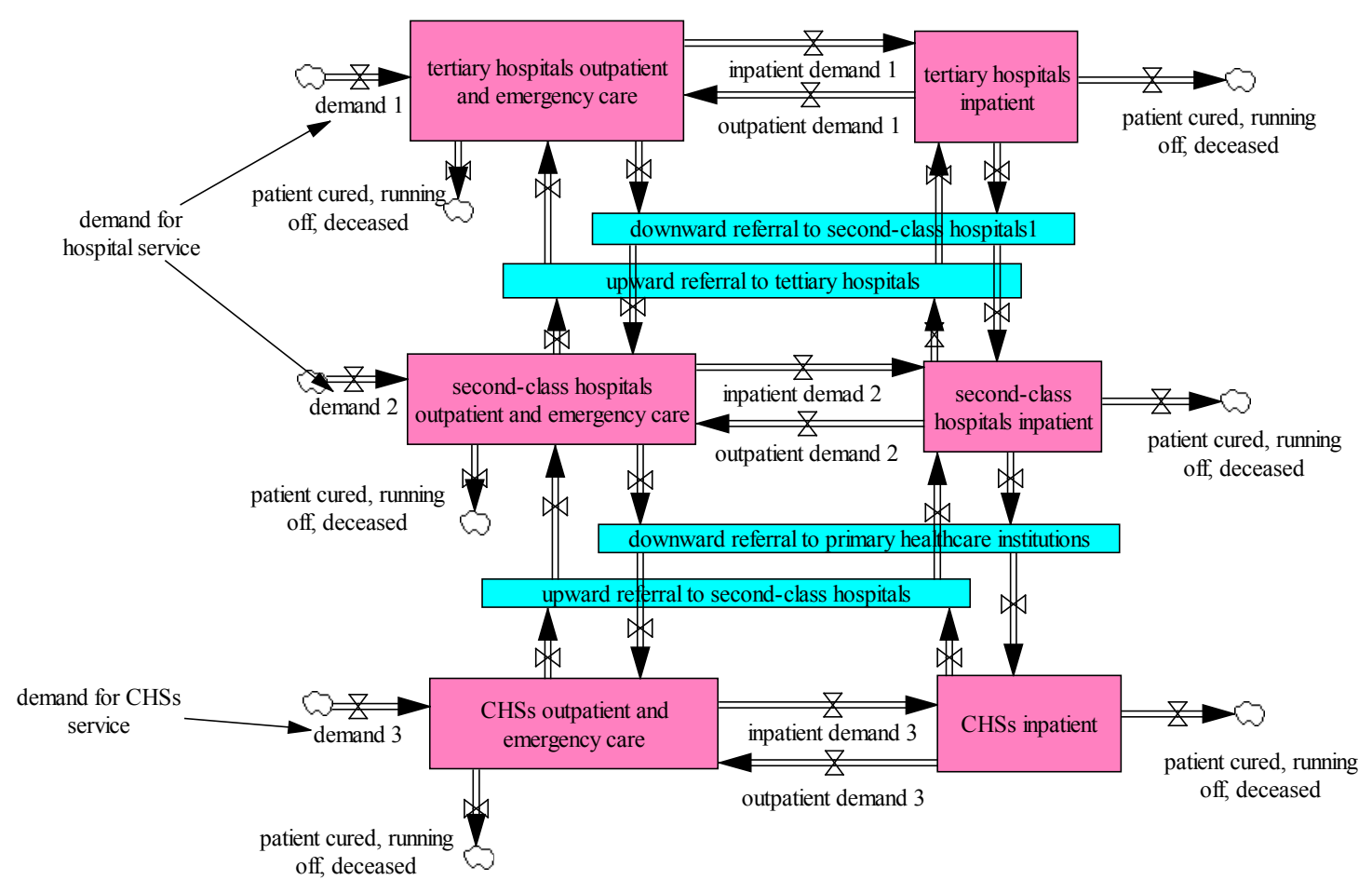

Figure 2: Stock-flow diagram of subsystem of service utilization in hospitals and CHSs. 


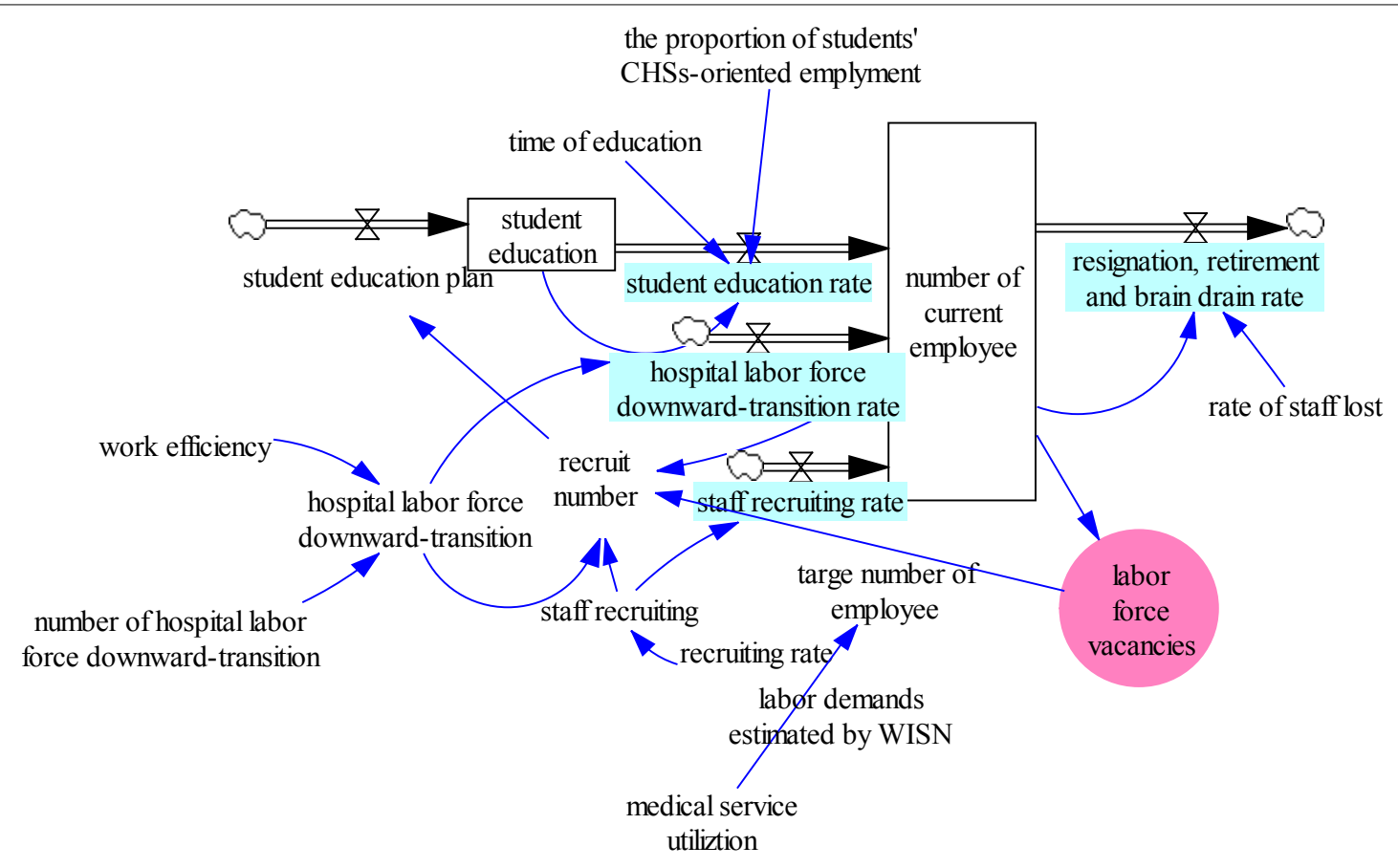

Figure 3: Stock-flow diagram of human resources.

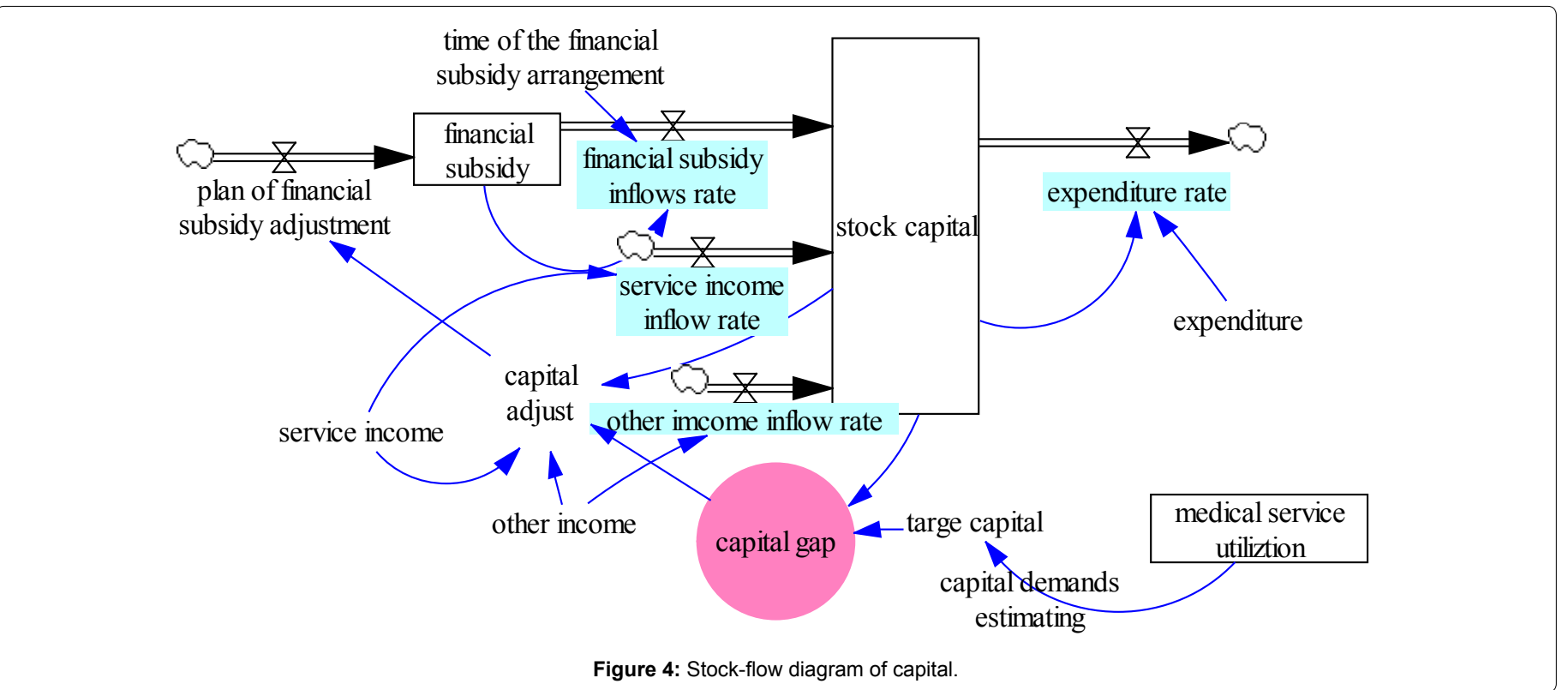

(2011-2015), compare the simulated data with actual data taken from historical records (the Zhejiang Health Statistical Yearbook, health care institution statistical reports). The mean error of outpatient utilization is $2.35 \%$, and that of inpatient is $1.07 \%$.

\section{Sensitivity analysis}

We compare outputs by changing input parameters, in order to assess the model sensitivity. The outpatient care utilization distribution is the key point requiring intervention in the reform, and the reform measures may influence the rate of CHSs primary diagnosis and the referral rate. Therefore, we adjust the level of
CHSs primary diagnosis rate, and then simulate the outpatient utilization distribution in tertiary hospitals, second-class hospitals, and CHSs (Figures 1 and 5).

Note: System of the real world without adjustment: CHSs primary diagnosis rate $=50.09 \%$.

Adjustment: CHSs primary diagnosis rate $=60 \%$;

Adjustment: CHSs primary diagnosis rate $=100 \%$;

$\mathrm{M}$ represents million outpatients. 
Citation: Zhang H, Liu Y, Yang Q, Gu S, Zhen X, et al. (2017) A System Dynamics Simulation Model of Hierarchical Medical Care System Reform in China. J Health Med Informat 8: 253. doi: 10.4172/2157-7420.1000253

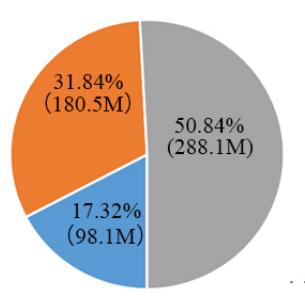

System - without adjustment Adjusted system 1
= tertiary hospital outpatient

- second-class hospital outpatient

$=$ CHSs outpatient

Figure 5: Sensitive analysis by adjusting CHSs primary diagnosis rate and referral rate.
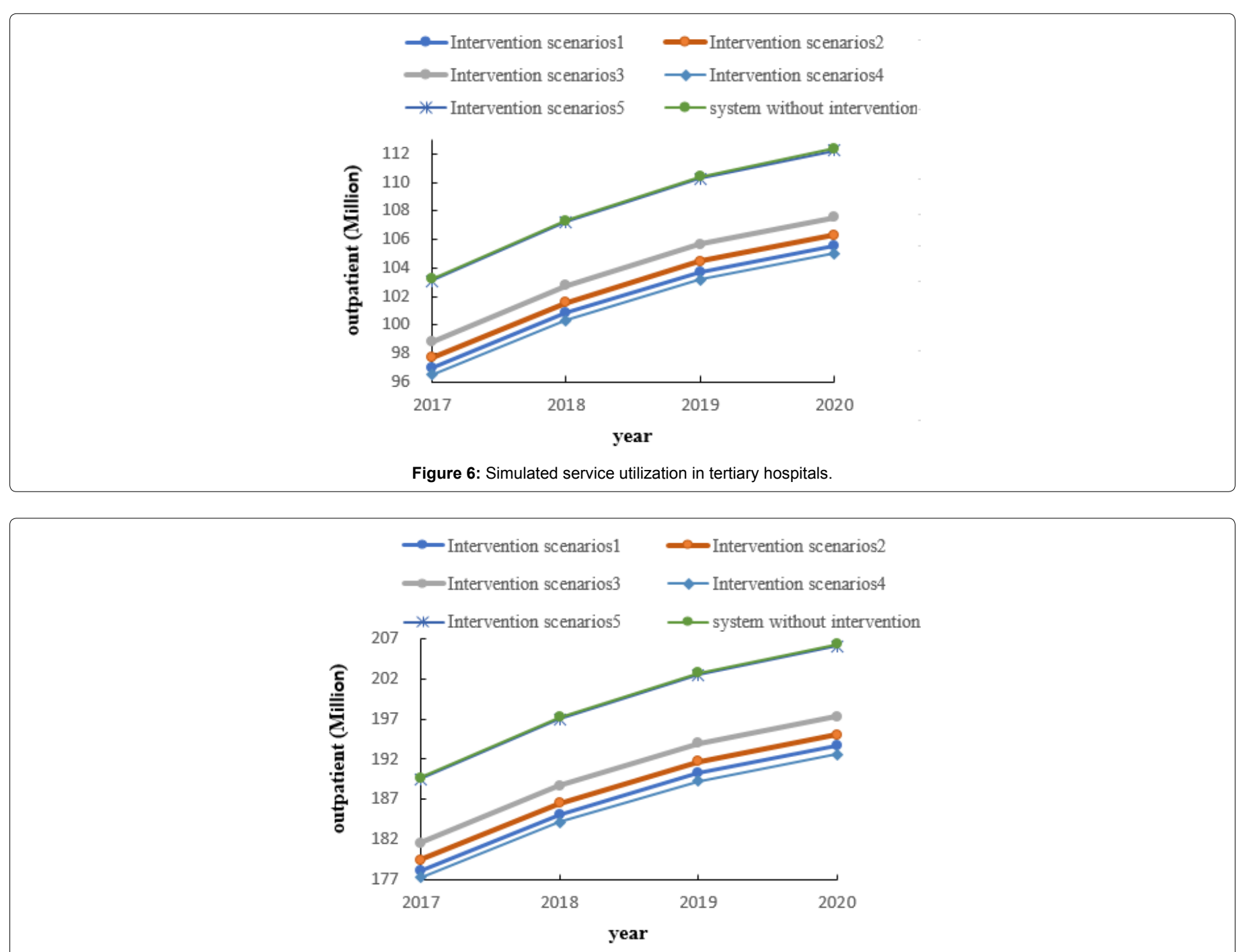

Figure 7: Simulated service utilization in second-class hospitals.

\section{Intervention scenarios}

Simulating the reform measure and the service utilization distribution are as shown in Figures 5-8.

Note: Intervention scenarios1: Improving medical devices and environment.
Intervention scenarios 2: Improving medical technology level.

Intervention scenarios 3: Improving medical care responsiveness.

Intervention scenarios 4: Decrease CHSs service price (The level of above-mentioned influencing factors is all divided to five grades, the intervention scenarios are: These factors optimized by one grade). 
Citation: Zhang H, Liu Y, Yang Q, Gu S, Zhen X, et al. (2017) A System Dynamics Simulation Model of Hierarchical Medical Care System Reform in China. J Health Med Informat 8: 253. doi: 10.4172/2157-7420.1000253

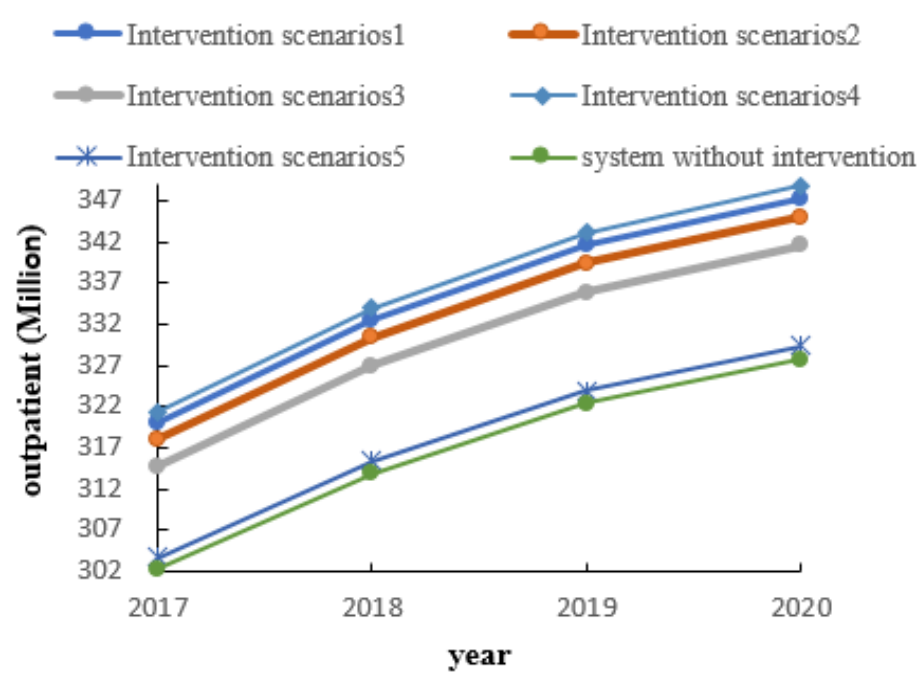

Figure 8: Simulated service utilization in CHSs.

Intervention scenarios 5: Increasing the gap of medical insurance compensable proportion in CHSs and hospitals by $10 \%$.

\section{Conclusion}

The SD tool is suitable to simulate hierarchical medical care system reform in china. A successful application of this approach will provide us with a visualized model of the medical care system, and will clearly illustrate of the internal interactions of factors and subsystems. The reliability of the model is verified by actual historical data tests and sensitivity analysis. Different scenarios by setting different levels of the input parameters may simulate the effects of reform measures. Reasonable resources allocation schemes are likely to get according the changes of utilization distribution*. Such tools can be readily scalable, applicable for complex system modelling, and has promising prospect for forecasting policy effect and optimizing policy measures.

The successful usage of this model provides us with a human resources allocation scheme and a cost savings list. We submitted the two articles involving these results to Chinese Journal of Health Policy.

\section{References}

1. Word Health Organization (2008) The World Health Report 2008: primary health care-now more than ever.

2. Word Health Organization (2000) The World Health Report 2000: improving performance.

3. Chen $Z$ (2009) Launch of the health-care reform plan in China. The Lance 373: $1322-1324$

4. Meng Q, Xu L, Zhang Y, Qian J, Cai M (2012) Trends in access to health services and financial protection in China between 2003 and 2011: a crosssectional study. The Lancet 379: 805-814.

5. Yu W, Li M, Ge Y, Li L, Zhanget Y, al. (2015) Transformation of potential medical demand in China: A system dynamics simulation model. J Biomed Informat 57: 399-414.

6. Brailsford S, Hilton $\mathrm{N}$ (2001). A comparison of discrete event simulation and system dynamics for modelling health care systems. Lancet 377: 641-649.

7. Merrill JA, Deegan M, Wilson RV, Kaushal R, Fredericks K (2013) A system dynamics evaluation model: implementation of health information exchange for public health reporting. J Am Med Informat Assoc 20: 131-138.

8. Fallah-Fini S, Rahmandad H, Terry TKH, Bures RM (2014) Modelling US adult obesity trends: a system dynamics model for estimating energy imbalance gap. American Journal of Public Health 104: 1230-1239.

9. Tobias MI, Cavana RY, Bloomfield A (2010). Application of a system dynamics model to inform investment in smoking cessation services in New Zealand. Am J Public Health 100: 1274-1281.

10. Schiehlen WO (2013) Advanced multibody system dynamics: Simulation and Software tools. Springer Science \& Business Media.

11. Xiao G (2011) Analysis of rural residents' willingness of getting care in township hospitals and its influence factors. Chinese Health Serv Manag 28: 854-855.

12. Dou WJ, Zhao F, Gu JL (2015) Survey of rural residents' wills to medical treatment and its influencing factors in Shandong province. Chinese $\mathrm{J}$ Gen Pract 13: 1484-1485.

13. Wu H, Ge CY, Jia HY (2011) The residents' visiting wills and service utilization in primary health care institutions. Chinese J Gen Pract 11: 491-492.

14. Chai L, Xu Z (2016) Forecast of China Population under Different Fertility Policy[J]. Open J Soc Sci 4: 213-215.

15. Yip W, Hsiao W, Chen W, Hu S, Ma J, et al. (2008) The Chinese health system at a crossroads. Health Affairs 27: 460-468.

16. Kleyn J, Arashi M, Bekker A, Millard S (2017) Preliminary testing of the CobbDouglas production function and related inferential issues. Communications in Statistics-Simulation and Computation 46: 469-488.

17. http://apps.who.int/iris/bitstream/10665/44414/1/9789241500197 users eng pdfhttp:/apps.who.int/iris/bitstream/10665/44414/1/9789241500197_users_ eng.pdf 